regions, $\mathrm{W}$ was deposited on the exposed gate, source, and drain regions. After the formation of an initial thin $\mathrm{W}$ film, a $\mathrm{SiH}_{4}$ reduction reaction took place with a deposition rate of $\sim 240 \mathrm{~nm} / \mathrm{min}$. The $\mathrm{WF}_{6} /$ $\mathrm{SiH}_{4}$ gas flow rate was kept at 40/12 and the process temperature was $300^{\circ} \mathrm{C}$. Conventional devices without $\mathrm{W}$ film deposition were also fabricated to serve as controls. A $500 \mathrm{~nm}$ Al film was deposited, patterned, and sintered at $400^{\circ} \mathrm{C}$ for $30 \mathrm{~min}$ to form metal pads. To reduce trap density and improve interface quality, wafers were also immersed in a $\mathrm{NH}_{3}$ plasma. The poly-Si gate was $10 \mu \mathrm{m}$ in length and $3 \mu \mathrm{m}$ in width. The oxide gate thickness was $\sim 30 \mathrm{~nm}$.

The researchers observed that the $\mathrm{W}$ TFTs have a larger driving current then conventional TFTs, especially under high gate bias. The parasitic resistance of the $\mathrm{W}$-TFTs is $\sim 4 \mathrm{k} \Omega$, which is three times smaller than that of conventional TFTs. In addition, the researchers found that a $\mathrm{NH}_{3}$ plasma treatment before the deposition of $\mathrm{W}$ contacts achieves a better inter- face quality and junction integrity of $\mathrm{W}$ TFTs than for $\mathrm{NH}_{3}$ plasma treatment after deposition of the $\mathrm{W}$ contacts. The researchers said this happens because the $\mathrm{W}$ blocks the plasma passivation from reaching the active regions under the contacts in the latter case.

MAXIM NIKIFOROV

\section{In Situ TEM Observations Reveal Growth Mechanisms for Carbon Nanofibers}

The synthesis of carbon nanotubes and nanofibers with specific structures and functionality is very important for nanotechnology applications. Carbon nanofibers are grown from the vapor phase in the presence of a catalyst. The atomicscale growth mechanisms for forming nanofibers have not been understood thus far. This understanding is crucial for manufacturing carbon nanofibers with tailored properties and characteristics. Now, S. Helveg at the Danish company Haldor Topsøe, F. Abild-Pedersen at the Technical University of Denmark, and their colleagues have used time-resolved, high-resolution in situ transmission electron microscopy (TEM) to elucidate the growth mechanisms of carbon nanofibers.

As the researchers reported in the January 29 issue of Nature, carbon nanofibers are formed by the decomposition of methane vapor at $500^{\circ} \mathrm{C}$ over a catalyst consisting of Ni nanoclusters with diameters of 5-20 nm supported on $\mathrm{MgAl}_{2} \mathrm{O}_{4}$. The growth experiments were performed in an in situ TEM, and a large number of consecutive real-time TEM images were obtained and stitched into a TEM movie. The nanofibers formed through a reactioninduced reshaping of the Ni nanocrystals into highly elongated shapes. The reshaping of the nanoclusters helped align the graphene sheets into tubular structures forming the nanofibers. The researchers said that this reshaping is abrupt, oscillating between spherical and elongated, but is critical for the formation of the fibers. The Ni nanoparticles were found to remain crystalline throughout the growth process. The nucleation and growth of the

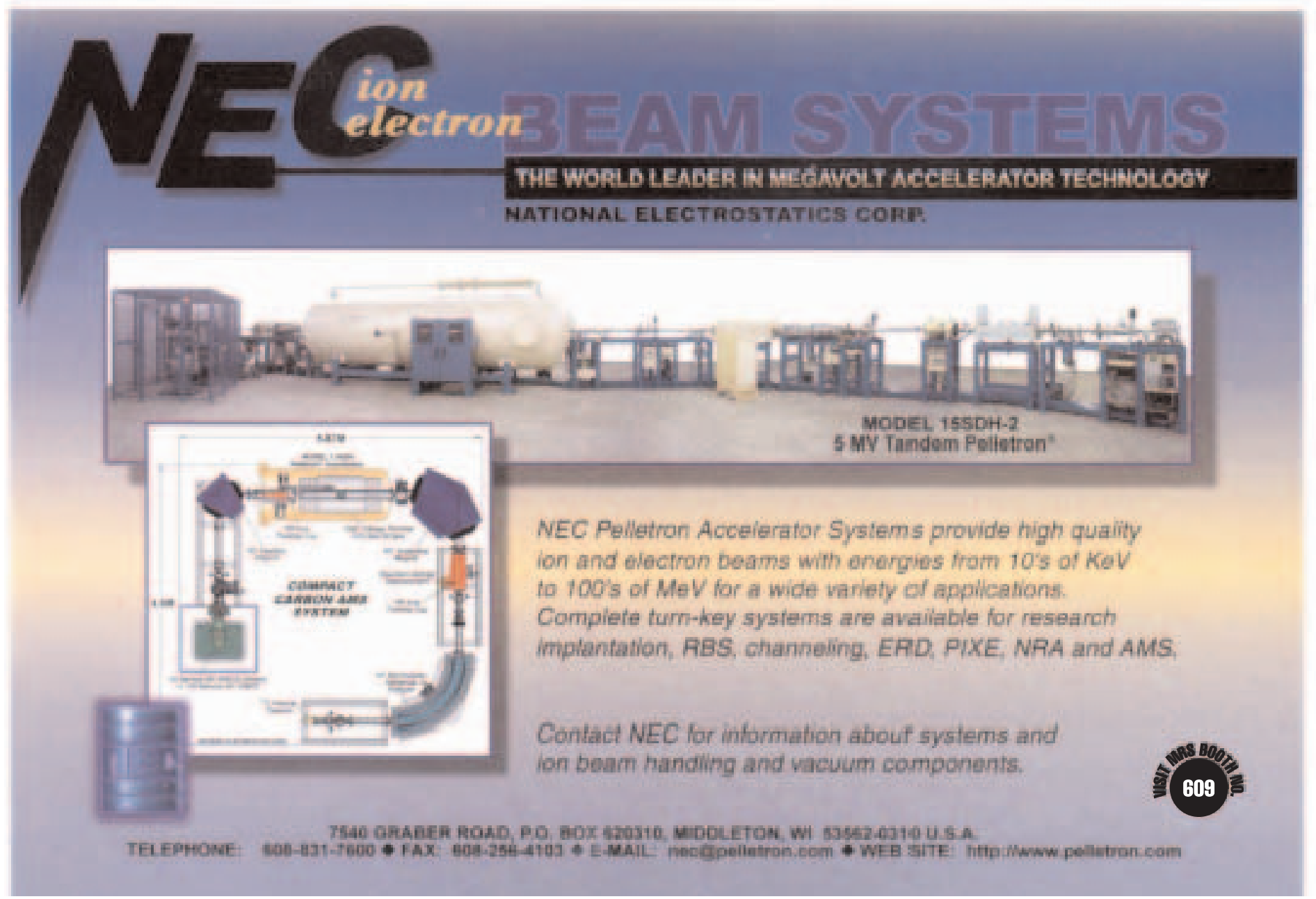

For more information, see http://advertisers.mrs.org 


\section{Thin Films: \\ No longer a hard problem!}

\section{Solutions from Hysitron}

Thin films of hard materials are a problem to characterize if you use a

"high load" instrument that has been modified for low load studies.

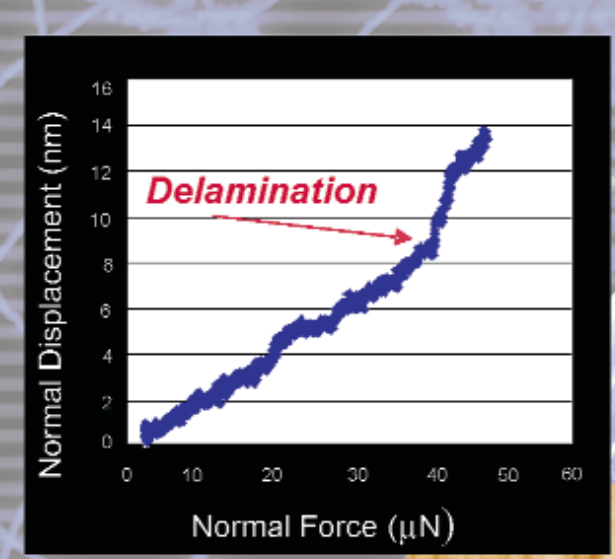

Delamination occurs on a computer hard disk surface at $40 \mu \mathrm{N}$ normal load

Ramp Load Scratch yields interfagial adhesion information. $10 \mu \mathrm{m}$ topographical image on a nanoscratch computer hard disk surface

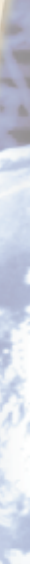

\section{nanoindentation instruments \\ The Hysitron family of} has been designed from the beginning for true
nanoscale studies. the beginning for true
nanoscale studies. Displacement-controlled indent on a nanocrystalline diamond film on a silicon substrate
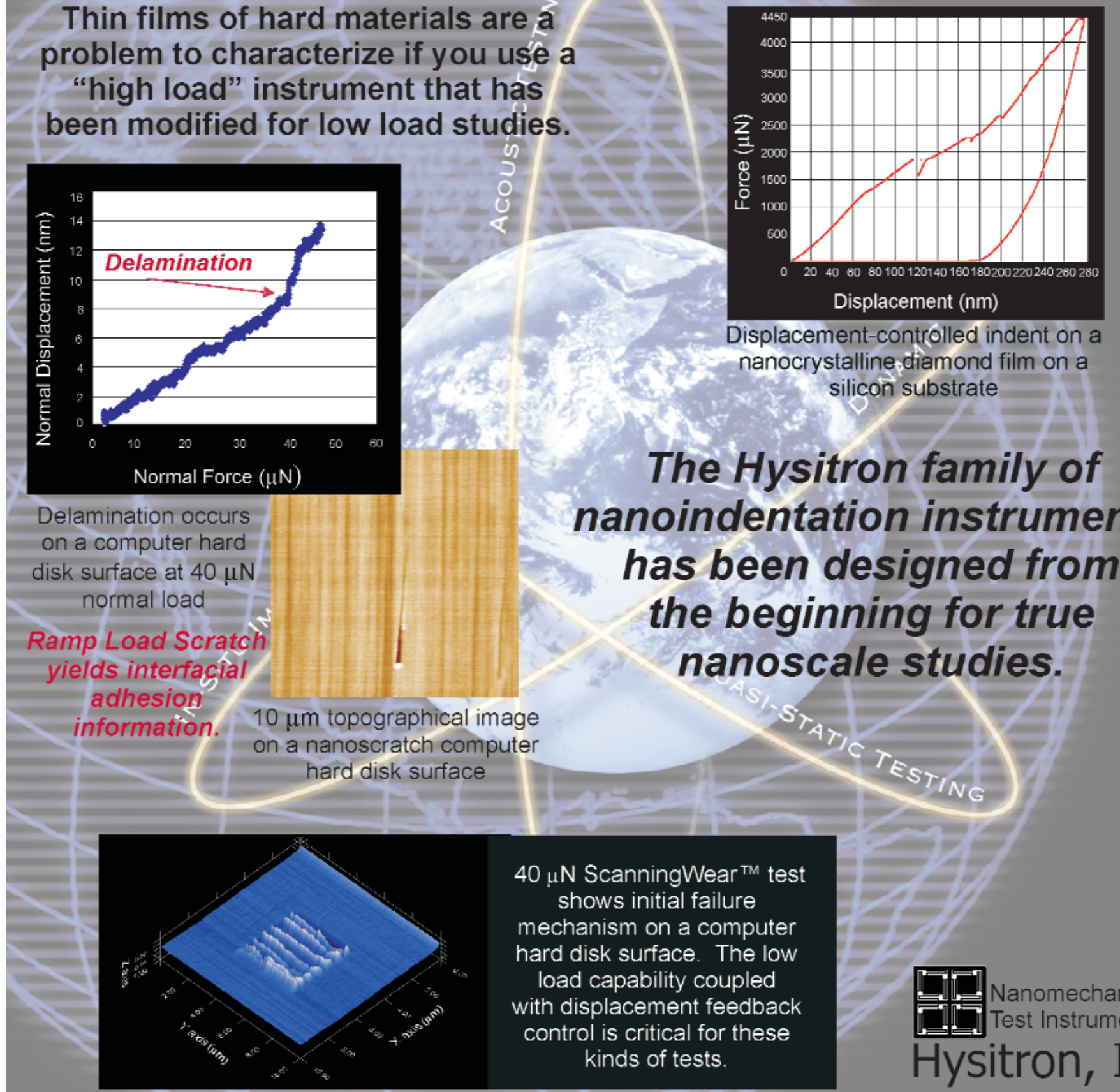


\section{www.gene.com}

\section{JOIN \\ GENENTECH!}

For over 28 years, Genentech has been at the forefront of the biotechnology industry, using human genetic information to discover, develop, commercialize and manufacture biotherapeutics that address significant unmet medical needs. Seventeen approved biotechnology products originate from or are based on our science, and Genentech manufactures and markets twelve products providing innovative treatments for cancer, heart disease, and respiratory and growth disorders. We continue our groundbreaking research to discover new therapies for lifethreatening medical conditions. Do the research to advance your career. Research Genentech.

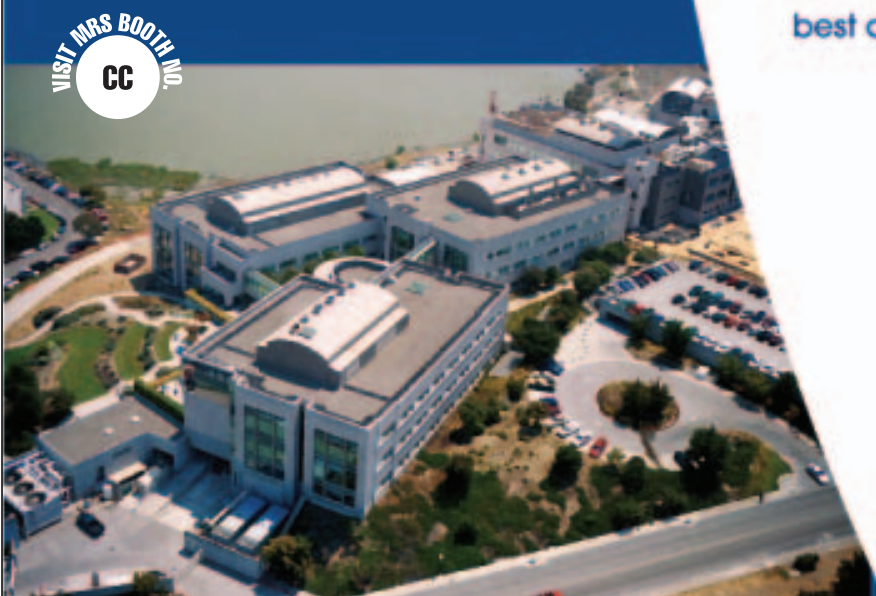

\section{JOIN THE RANKS OF BIOTECH'S BEST!}

An award-winning employer, Genentech demands the best from its employees. In return, our employees are rewarded with familyfriendly benefits and programs that are among the best in the industry, including stock options, a paid sabbatical program, and one of the largest corporate-sponsored childcare centers in the country.

Our company is growing and we are seeking

talented candidates to join us in the following opportunities at our South San Francisco and Vacaville locations:

- Chemistry

- ENGINEERING

- facilities

- global SUPPIY ChaIN MANAgEMENT

- manufacturing

- QA/QC

- regulatory

Move to an environment that aftracts, retains and rewards the best and brightest employees in all areas. Join Genentech!

To view these and all our career opportunities, please visit us online at:

www.gene.com/careers

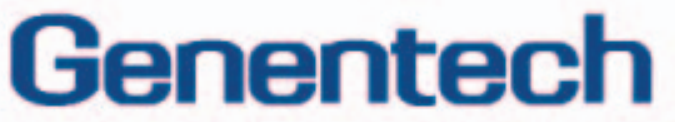

IN BUSINESS FOR LIFE

We are an Equal Opportunity Employer. 
graphene layers, which eventually form the nanofibers, were assisted by dynamic formation of monoatomic step ledges on the graphene- $\mathrm{Ni}$ interface.

The researchers performed density functional theory calculations to understand the origins of the interfacial process and the transport of $\mathrm{C}$ and $\mathrm{Ni}$ atoms along the graphene-Ni interface. The surface transport of the $\mathrm{C}$ atoms was shown to be the rate-limiting step for the nanofiber growth. The experimental results and theoretical calculations indicate that the step edges on the Ni surface act as growth centers for graphene growth because $\mathrm{C}$ binds more strongly to such sites. This mechanism is likely to be of broad significance for any metal-catalyzed nanofiber growth, according to the researchers. They said that this study reveals the first direct glimpses of the initial stages of nanofiber growth using nanoscale real-time growth observations. Metallic step-edges acting as spatio-temporal dynamic growth sites may be important for understanding other types of catalytic reactions and nanomaterial syntheses.

\section{GOPAL RAO}

\section{Variation of Electron Level Enables Semiconducting-to-Metallic Transition in Single Phenalenyl- Based Molecules}

Quantum transport properties of singlemolecule electronic devices are affected not only by the choice of molecules but also by the interface structures used. K. Tagami and M. Tsukada from the University of Tokyo and L. Wang from Southern Yangtze University in China studied theoretically the conductive properties of single boron- and nitrogensubstituted phenalenyl molecules $\left(\mathrm{C}_{13} \mathrm{H}_{9}\right)$ and found that they can tune the properties of the molecules from semiconducting to metallic depending on the type of central atom used. The researchers reported in the February issue of Nano Letters that by changing the type of central atom from carbon to nitrogen or boron, they can dramatically alter the transport properties of the system.

The researchers calculated the atomic coordinates (i.e., the size) of each molecule, and connected it to electrodes by bonding a mercapto-vinyl group to the $\alpha$ or $\beta$ site, which is the second- and thirdnearest to the central atom site, respectively. Molecules connected through $\beta$ sites demonstrate a conduction channel that is energetically very close to the Fermi energy of the gold electrodes. In contrast, with the connection through the $\beta$ site, nitrogen- and carbon-containing phenalenyl molecules become semicon- ducting, while the boron-type displays metallic properties. The researchers also found that when the electrodes are connected to the $\beta$ sites, the conductance through the carbon-containing phenalenyl molecule is spin-dependent. The researchers said that the change in the transport features originates from the spatial distribution of the nonbonding molecular orbital, which has amplitude only on the $\alpha$ sites. These features do not change when the researchers substitute the central atom of phenalenyl with the nitrogen; however, if they substitute it with the boron atom, then the quantum transport occurs through the orbitals that have amplitude both on $\alpha$ and $\beta$ atomic sites.

EKATERINA A. LITVINOVA

\section{Stationary Light Pulses Created}

Achieving direct control over the propagation of light is a goal that is interesting both from a fundamental scientific standpoint and for practical applications. If developed, the ability to control light pulses may lead the way to new advances in nonlinear optics and quantum computing. Recently, a team of physicists from Harvard University and Lebedev Institute of Physics, Moscow, temporarily stored light pulses as electromagnetic energy. Previous research efforts have stored localized light pulses as holographic imprints, but this work improves on those studies by actually trapping photons in an atomic medium.

As reported in the December 11, 2003, issue of Nature, M. Bajcsy, A.S. Zibrov, and M.D. Lukin designed a technique for the creation, storage, and controlled release of stationary light pulses. The main concept is based on the principle of electromagnetically induced transparency (EIT), which uses one or more "control" laser beams to modify the optical properties of a medium. The methods described in this work employ a weak "signal" laser beam containing the photons that will be stopped, and two control lasers: a forward-directed (FD) beam and a backward-directed (BD) beam.

The experimental realization of this idea is carried out using an optically dense medium of $\mathrm{Rb}$ vapor contained in a small cell and open to the appropriate laser beams. The group first maps a signal pulse onto the $\mathrm{Rb}$ by firing a signal pulse at the Rb gas as it is illuminated by the FD control beam, similar to previous EIT experiments. Turning off the FD beam stores the signal on the $\mathrm{Rb}$ atoms, and subsequently turning on either of the control beams converts the stored excitation back into light, propagating in the direction of the applied beam. The
S

3D MODELING solutions

S3D Modeling software is a unique scientific tool for 3-dimensional modeling of granular media.

S3D Modeling product family provides comprehensive visual analysis and wide range of quantitive characteristics of the granular structures.

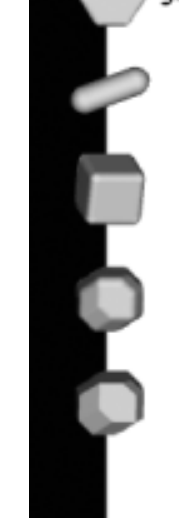

Nanofilms

Coatings

Powders

Ceramics

Composites

Refractories *.*

Grain structure

Pore structure ....

Packing

Sintering

and more.

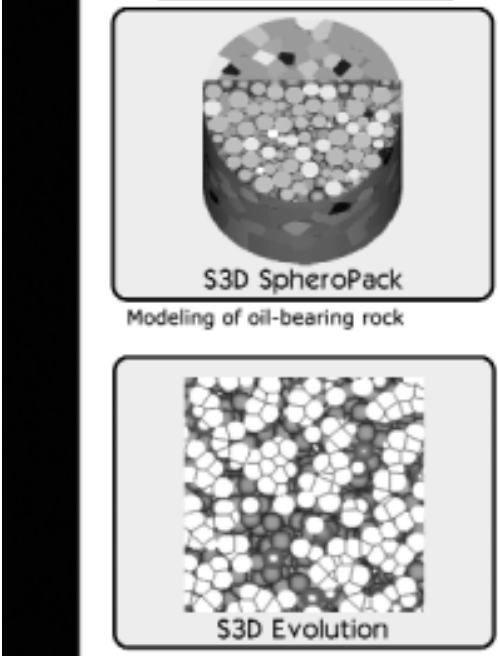

Modeling zonal segregation during the disperse powders sintering

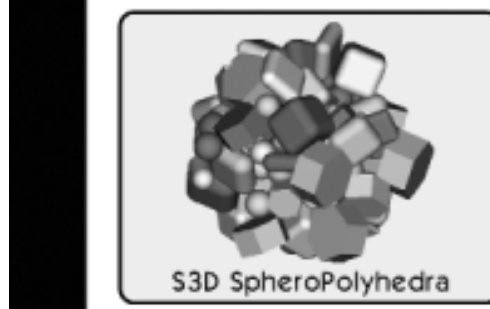

S3D SpheroPolyhedra

Multiphase polydisperse centra packing of particles with varied morphology

www.smartimtech.com

(9) SMART IMAGING TECHNOLOGES Tel: US: (877) 280.11.00 (toll-froe) Tel: US: $(877) 280.11 .00$ (toll-free)
Tel: International +1 (713) 589.32 .16 E-mait: infoesarmartimbech.com

For more information, see http://advertisers.mrs.org 